\title{
Wavefront-sensor-based electron density measurements for laser-plasma accelerators
}

\author{
G. R. Plateau,* N. H. Matlis, C. G. R. Geddes, A. J. Gonsalves, \\ S. Shiraishi, C. Lin, R. A. van Mourik, and W. P. Leemans ${ }^{\dagger}$ \\ LOASIS program, Lawrence Berkeley National Laboratory (LBNL) \\ 1 Cyclotron Road, Berkeley, 94720 CA, USA
}

(Dated: March 18, 2010)

\begin{abstract}
Characterization of the electron density in laser produced plasmas is presented using direct wavefront analysis of a probe laser beam. The performance of a laser-driven plasma-wakefield accelerator depends on the plasma wavelength, hence on the electron density. Density measurements using a conventional folded-wave interferometer and using a commercial wavefront sensor are compared for different regimes of the laser-plasma accelerator. It is shown that direct wavefront measurements agree with interferometric measurements and, because of the robustness of the compact commercial device, have greater phase sensitivity, straightforward analysis, improving shot-to-shot plasma-density diagnostics.

PACS numbers: 52.70.Kz, 07.60.Ly, 52.38.-r, 52.25.Jm, 52.35.Tc
\end{abstract}

\footnotetext{
*Also at École Polytechnique, Palaiseau, France; Electronic address: grplateau@lbl.gov

$\dagger$ Electronic address: wpleemans@lbl.gov
} 


\section{INTRODUCTION}

Laser-plasma accelerators (LPA) [1-4] rely on the excitation of an electron density wave by a laser in a plasma. The electron density, $n_{e}$, determines key parameters of the accelerator such as the dephasing length, the pump depletion length, and the maximum amplitude of a nonlinear plasma wave [5]. The present generation of LPAs is being developed to serve as a unique source for generating $\mathrm{THz}$ and x-ray light [6-8]. The performance of such light sources is determined in particular by the plasma shape and density. For instance, the radiated energy and duration of ultrashort $\mathrm{THz}$ pulses produced by accelerated electron bunches crossing the plasma-vacuum boundary (coherent transition radiation), depends on the sharpness of the transition and on the transverse size of the plasma [9-11]. Mapping the electron density of the plasma is therefore necessary to understand the $\mathrm{THz}$ generation mechanism. In betatron based x-ray sources, the x-ray energy is in part determined by the plasma density [12-14].

Plasma density measurements are conventionally performed using non-perturbative laser interferometric techniques (Michelson, Mach-Zender configurations). In these techniques a laser beam, usually a short $(<1 \mathrm{ps})$ pulse, is split and propagates along two beam paths. In one arm the laser pulse goes through the plasma and experiences a phase shift due to a local variation of the refractive index. By interfering the laser pulse from this arm with the laser pulse in the other arm, called the reference arm, the relative phase is retrieved by Fourier analysis. The electron density is finally deduced from the phase map via its relation to the refractive index [15]. For most interferometers, the reference and probe laser pulses travel along significantly different paths, and effects such as vibration of the optics can cause shot-to-shot change in the relative phase. This increases the noise in the measurement. In this paper, an alternative technique [16-19] using a wavefront sensor is demonstrated in which only one laser pulse is required. Several types of wavefront sensors are commercially available (Hartmann, Shack-Hartmann, shearing interferometer). The setup used for both folded-wave interferometry and wavefront sensing is described in Sec. II A. Analysis and density map reconstruction are discussed in Sec. II B. Electron density measurements using this new technique were benchmarked with interferometric measurements for a range of plasma densities (Sec. III) and the ability to resolve strong density gradients was successfully demonstrated. Furthermore, it is shown that for the setup presented in this paper 
phase sensitivity and hence accuracy in determining the electron density can be significantly improved by using a wavefront sensor.

\section{ELECTRON DENSITY MAP RECONSTRUCTION}

The experiments were performed using a laser-driven plasma-wakefield accelerator in the self-modulated regime [20, 21] relying on self-trapping of background electrons. A laser pulse of central wavelength $800 \mathrm{~nm}(\geqslant 40 \mathrm{fs}$, up to $0.5 \mathrm{~J})$ was focused $\left(w_{0} \simeq 6 \mu \mathrm{m},>\right.$ $10^{19} \mathrm{~W} / \mathrm{cm}^{2}$ ) into Helium or Hydrogen supersonic gas from a supersonic nozzle [22]. The

focus was $1 \mathrm{~mm}$ above the nozzle. The laser pulse excited a plasma density wave which trapped and accelerated up to 10's of MeV electron bunches with $\sim 1 \mathrm{nC}$ of charge. Typical electron densities were on the order of $3 \cdot 10^{19}$ electrons per cubic centimeter $\left(e^{-} / \mathrm{cm}^{3}\right)$, which corresponds to a plasma wavelength of $\lambda_{p} \simeq 6 \mu \mathrm{m}$. Interferometric measurements are possible at these densities using wavelengths shorter than $\sim 6 \mu \mathrm{m}$. In these experiments transverse interferometry was carried out using a laser pulse of central wavelength $400 \mathrm{~nm}$ and 70 fs FWHM duration.

\section{A. Experimental setup}

Both a wavefront sensor and a folded-wave interferometer [23] were used to characterize the electron density of the plasma. In the folded-wave interferometer, the lower part of a probe beam, which has a transverse size large compared to the plasma diameter, passed through the plasma. After passing through the plasma, the probe beam was split into two laser beams of equal intensity. By spatially inverting the beam in one arm before recombining the two beams, the area of each laser beam unaffected by the plasma interfered with the affected area of the other (Fig. 1a). Each arm therefore served as the reference of the other.

The setup with wavefront sensor is shown in figure $1 \mathrm{~b}$. The sensor measures directly the phase front curvature of an incoming laser beam and therefore does not require the foldedwave interferometer. The amount of phase introduced in the laser beam passing through the plasma is retrieved by subtracting a reference phase map obtained when the plasma is absent. 


\section{B. Electron density reconstruction}

Wavefront sensor and interferometric measurements use different algorithms to recover the phase information. The wavefront sensor used in these experiments was a commercial 4-wave shearing interferometer. Measurements are based on a modified Hartmann test [24], in which diffraction-based limitations are greatly reduced by adding a phase chessboard to the classical Hartmann mask. A classical Hartmann test uses a mask of holes splitting the incoming light into beams whose deflections are proportional to the local distortions of the analyzed wavefront. By adding a second mask a 2D diffraction grating is created, which replicates the incoming beam into 4 identical waves propagating along different directions. A Fourier analysis of the interference grid allows reconstruction of the phase gradient in 2 orthogonal directions. The phase map is obtained by integration of these gradients. The phase recovery routine is provided by the manufacturer.

Using the folded-wave interferometer, the plasma density was recovered from the interferograms by fringe pattern analysis [25-27]. A fast-Fourier-transform (FFT) was applied line-by-line on the interferograms (Fig. 2, left). Filtering out the carrier frequency and computing the inverse Fourier transform, the phase information was retrieved as the phase of the complex space-domain signal (Fig. 2, center).

The fringe pattern of a folded-wave interferogram has the form $f(x, y)=a(x, y)+$ $b(x, y) \cos \left[2 \pi f_{0} x+\phi(x, y)\right]$ where $a(x, y)$ and $b(x, y)$ are due to uniformities of the intensity profile in the probe beam, $\phi(x, y)$ is the phase difference due to the presence of the plasma and $f_{0}$ is the spatial-carrier frequency. In complex notations the fringe pattern can be written $f(x, y)=a(x, y)+c(x, y) \exp \left(2 \pi j f_{0} x\right)+c^{*}(x, y) \exp \left(-2 \pi j f_{0} x\right)$ where * denotes the complex conjugate and $c(x, y)=1 / 2 \cdot b(x, y) \exp [j \phi(x, y)]$. A FFT of this equation yields $F(f, y)=A(f, y)+C\left(f-f_{0}, y\right)+C^{*}\left(f+f_{0}, y\right)$. The phase information is simply

retrieved as the argument of the inverse-FFT of the term $C\left(f-f_{0}, y\right), \mathfrak{F}^{-1}\left[C\left(f-f_{0}, y\right)\right]=$ $1 / 2 \cdot b(x, y) \exp \left\{j\left[\phi(x, y)+2 \pi j f_{0} x\right]\right\}$. A linear fit on an unperturbed part of the interferogram provides $f_{0}$ which contribution can then be subtracted. The phase information is retrieved within $[-\pi ; \pi]$ and to avoid any non-physical discontinuities the phase map needs to be "unwrapped". When the difference between two adjacent values along the horizontal axis exceeds $\pi$ it is compensated. The formula used for these experiments is: $\phi_{\text {unwrapped }}(0)=\phi(0)$ and $\forall i \in\{1 ; \ldots ; n-1\}, \phi_{\text {unwrapped }}(i)=\phi(i)-2 \pi \times\lfloor 1 / 2+(\phi(i)-\phi(i-1)) / 2 \pi\rfloor$ where $n$ is 
the number of pixels on the axis. The unwrapping is applied on each line and each column of the phase map.

For both diagnostics the electron density map (Fig. 2, right) was computed, using its relation to the plasma refractive index of refraction, by an Abel inversion [15, 28]. For a non-magnetic plasma and in the absence of a relativistically intense laser pulse, the refractive index is given by $\eta_{p}^{2}=1-\omega_{p}^{2} / \omega^{2}=1-n_{e} / n_{c}(\omega)$ where $\omega$ is the angular frequency of the probe

beam and $n_{c}(\omega)=\epsilon_{0} m_{e} \omega^{2} / e^{2}$ is the critical density. For $n_{e}<n_{c}$ and $\eta_{\text {gas }} \simeq 1$, the phase lag between reference and probe is then $\phi=\int\left(1-\eta_{p}(\omega)\right) \omega / c d l$, where the integral is performed along the beam path in the plasma and $c$ is the vacuum speed of light. Substituting the definition of $\eta_{p}$ in this equation yields $\phi(x, y)=\omega / c \int\left(1-\sqrt{1-n_{e}(x, y) / n_{c}(\omega)}\right) d l$. Here, the phase is a measure of the average refractive index along the path in the plasma. Assuming the plasma is cylindrically symmetric, the measured phase is therefore an Abel transform of the actual physical quantity. After symmetrization of the unwrapped phase map, using the vertical location of its center of mass as axis of symmetry (Fig. 2, right), an Abel inversion is computed, $\Phi(x, r)=-1 / \pi \int_{r}^{R} \partial \phi(x, y) / \partial y \cdot\left(y^{2}-r^{2}\right)^{-1 / 2} d y$, where $\phi(x, R)=0$. From the unwrapped and Abel inverted phase map, the electron density of the plasma can be calculated by inverting the previously established relation between phase and density, $n_{e}(x, r)=n_{c}(\omega)\left[1-(1-c / \omega \cdot \Phi(x, r))^{2}\right]$. For both phase maps retrieved from folded-wave interferometry and wavefront sensing, symmetrization, Abel inversion, and conversion to electron density were computed. In the next section, the difference between the two types of measurements is studied.

\section{PLASMA DENSITY MEASUREMENTS USING A WAVEFRONT SENSOR}

Measurements were performed for different back pressures of the gas jet, namely 500 psi, 600 psi, and 700 psi Hydrogen. For each of these pressures, wavefront-sensor-based measurements and folded-wave interferograms were alternatively taken. A mean phase map of over 50 pictures was computed for both types of measurements. Both mean phase maps were then symmetrized, Abel inverted, and converted to electron density according to the equations presented in Sec. IIB. Analysis shows good agreement between the two types of measurements. As an example, the contour plots of the mean phase maps and mean density maps at 600 psi Hydrogen are compared in Fig. 3. The two contours of density in 
Fig. 3b differ from each other near the symmetry axis. This difference is attributed to the Abel inversion, which is sensitive to noise close to the symmetry axis since the integration $\int_{r}^{R} 1 / \sqrt{\left(y^{2}-r^{2}\right)} d y$ diverges for $r \simeq 0$. A discrepancy between the phase maps is also observed for higher phase shifts (Fig. 3a) ranging from $6 \%$ at the center of the plasma to $22 \%$ around $z \simeq 1,1.8$ and $3 \mathrm{~mm}$ (Fig. 4). The difference in density measurements ranges from $6 \%$ to $17 \%$ in the center of the plasma and increases at low densities where the signal-to-noise is small $(\sim 1)$.

In addition, the ability to resolve strong density gradients was tested using a damaged gas jet nozzle which produced a strongly perturbed gas flow for Helium gas. Both measurements provide similar resolution of the perturbed density profile (Fig. 5).

In order to compare the scaling laws of the two techniques, the plasma density was analyzed as a function of gas pressure. Averages of the density maps were calculated over the plateau region where the density is nearly flat, excluding the zone near the axis where the Abel inversion fails. The shot-to-shot errors are dominated by fluctuations in gas flow (Table I). The in-quadrature contribution of the instrument resolution to the rms deviations is less than $4.4 \%$ for the wavefront sensor and $33 \%$ for the folded-wave interferometer (Fig. 6).

The phase sensitivity of both techniques was evaluated by measuring 188 consecutive phase maps in the absence of plasma and under the same experimental conditions. A rms deviation phase map was calculated for both types of measurement (Fig. 6). The averages of the maps are $95.7 \mathrm{mrad}$ and $11.4 \mathrm{mrad}$ for respectively the folded-wave interferometer and the wavefront sensor, making the wavefront sensor-based technique $\simeq 8.4$ times more sensitive. In addition, fluctuations over the phase maps are more homogeneous for the wavefront sensor measurements.

The spatial resolution of the diagnostic is determined by the intrinsic camera resolution and the magnification of the imaging system. In this paper the wavefront sensor camera was used for both types of measurements to avoid ambiguity in the interpretation of the images. It has $480 \times 640$ pixels of $7.5 \mu \mathrm{m}$ for both dimensions. Because the wavefront sensor is a 4 -wave shearing interferometer the size of a measurement point does not correspond to a pixel. The wavefront sensor produces intensity and phase maps of $120 \times 160$ measurements points with a spatial resolution of $29.6 \mu \mathrm{m}$ for both dimensions. Whereas the wavefront sensor has a fixed CCD chip and pixel size chosen by the manufacturer, it is in principle possible to choose a different camera to increase resolution of the folded-wave interferometer. 
Plasmas produced by the laser-gas interaction were typically $2 \mathrm{~mm}$ long and had a diameter of $0.2 \mathrm{~mm}$. After imaging the plasma to a primary focus shortly after the beam combiner (Fig. 1) with a $f / 7$ achromat lens, an imaging system using aspherical and cylindrical optics was used to provide higher resolution in the vertical direction to the wavefront sensor, $21.3 \mu \mathrm{m}$ per measurement point in the horizontal plane and $4.8 \mu \mathrm{m}$ per point in the vertical plane.

\section{CONCLUSION}

A simple single-shot wavefront-sensor-based electron density diagnostic is presented that relies on the use of a wavefront sensor. The design requires only one arm of a nonperturbative probe laser beam. Post-analysis requires only the computation of an Abel inversion. Successful resolution tests were performed by comparing wavefront sensing and folded-wave interferometry-based measurements for different pressures, thus electron densities and, for steep density gradients. The technique, which can be used for any phase sensitive measurement, was tested at the LOASIS facility at LBNL and found to provide the same information as a regular interferometer with improved phase noise and with greater ease of operation.

\section{ACKNOWLEDGMENTS}

The authors acknowledge Kei Nakamura, Csaba Tóth, Carl B. Schroeder, Estelle CormierMichel and Eric Esarey for their contributions.

This work is supported by the Director, Office of Science, High Energy Physics, U.S. Dept. of Energy under Contract no. DE-AC02-05CH11231 and DARPA. 
[1] T. Tajima and J. M. Dawson, Phys. Rev. Lett. 43, 267 (1979).

[2] E. Esarey, P. Sprangle, J. Krall, and A. Ting, IEEE Trans. Plasma Sci. 24, 252 (1996).

[3] C. G. R. Geddes, Cs. Tóth, J. van Tilborg, E. Esarey, C. B. Schroeder, D. Bruhwiler, C. Nieter, J. Cary, and W. P. Leemans, Nature 431, 538 (2004).

[4] W. P. Leemans, B. Nagler, A. J. Gonsalves, Cs. Tóth, K. Nakamura, C. G. R. Geddes, E. Esarey, C. B. Schroeder, and S. M. Hooker, Nature Physics 2, 696 (2006).

[5] E. Esarey, C. B. Schroeder, and W. P. Leemans, Rev. Mod. Phys. 81, 1229 (2009).

[6] W. P. Leemans, C. G. R. Geddes, J. Faure, Cs. Tóth, J. van Tilborg, C. B. Schroeder, E. Esarey, G. Fubiani, D. Auerbach, B. Marcelis, M. A. Carnahan, R. A. Kaindl, J. Byrd, and M. C. Martin, Phys. Rev. Lett. 91, 074802 (2003).

[7] W. P. Leemans, E. Esarey, J. van Tilborg, P. A. Michel, C. B. Schroeder, Cs. Tóth, C. G. R. Geddes, and B. A. Shadwick, IEEE Trans. Plasma Sci. 33, 8 (2005).

[8] P. Catravas, E. Esarey, and W. P. Leemans, Meas. Sci. Tech. 12, 1828 (2001).

[9] V. L. Ginzburg and I. M. Frank, Zh. Eksp. Teor. Fiz. 16, 15 (1946).

[10] J. V. Lepore and R. J. Riddell, Jr., Phys. Rev. D 13, 2300 (1976).

[11] C. B. Schroeder, E. Esarey, J. van Tilborg, and W. P. Leemans, Phys. Rev. E 69, 016501 (2004).

[12] E. Esarey, B. A. Shadwick, P. Catravas, and W. P. Leemans, Phys. Rev. E 65, 056505 (2002).

[13] A. Rousse, K. T. Phuoc, R. Shah, A. Pukhov, E. Lefebvre, V. Malka, S. Kiselev, F. Burgy, J.-P. Rousseau, D. Umstadter, and D. Hulin, Phys. Rev. Lett. 93, 135005 (2004).

[14] K. T. Phuoc, F. Burgy, J.-P. Rousseau, V. Malka, A. Rousse, R. Shah, D. Umstadter, A. Pukhov, and S. Kiselev, Phys. of Plasmas 12, 023101 (2005).

[15] I. H. Hutchinson, Principles of Plasma Diagnostics, 2nd ed. (Cambridge University Press, 84 Theobald's Road London WC1X 8RR UK, 2002).

[16] B. M. Welsh, B. L. Ellerbroek, M. C. Roggemann, and T. L. Pennington, Appl. Opt. 34, 21, 4186 (1995).

[17] K. L. Baker, J. Brase, M. Kartz, S. S. Olivier, B. Sawvel, and J. Tucker, Rev. Sci. Inst. 73, $11,3784(2002)$.

[18] T. Fukuchi, Y. Yamaguchi, T. Nayuki, K. Nemoto, and K. Uchino, Elec. Eng. in Japan 146, 
4, 10 (2004).

[19] N. Qi, R. R. Prasad, K. Campbell, P. Coleman, M. Krishnan, B. V. Weber, S. J. Stephanakis, and D. Mosher, Rev. Sci. Inst. 75, 10, 3442 (2004).

[20] N. E. Andreev, L. M. Gorbunov, V. I. Kirsanov, A. A. Pogosova, and R. R. Ramazashvili, Pis'ma Zh. Eksp. Teor. Fiz. 55, 551 (1992).

[21] E. Esarey, J. Krall, and P. Sprangle, Phys. Rev. Lett. 72, 2887 (1994).

[22] C. G. R. Geddes, K. Nakamura, G. R. Plateau, Cs. Tóth, E. Cormier-Michel, E. Esarey, C. B. Schroeder, J. R. Cary, and W. P. Leemans, Phys. Rev. Lett. 100, 215004 (2008).

[23] F. Martin, Appl. Opt. 19, 4230 (1980).

[24] J. Primot and N. Guérineau, Appl. Opt. 39, 5715 (2000).

[25] M. Takeda, H. Ina, and S. Kobayashi, J. Opt. Soc. Am. 72, 156 (1982).

[26] W. W. Macy, Appl. Opt. 22, 3898 (1983).

[27] P. Gao, B. Yao, J. Han, L. Chen, Y. Wang, and M. Lei, Appl. Opt. 47, 2760 (2008).

[28] M. Kalal and K. A. Nugent, Appl. Opt. 27, 1956 (1988). 

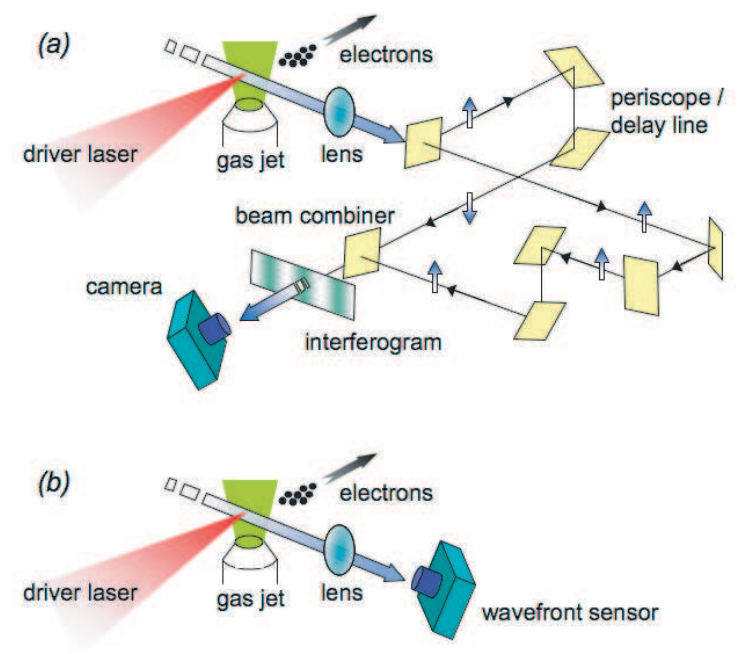

FIG. 1: Schematic of the plasma density diagnostics. When using the folded-wave interferometer the wavefront sensor is operated as a camera, both arms of the interferometer are used and interferograms are recorded (a). When using the wavefront sensor for phase front measurements of the probe beam only one arm is used (b). 

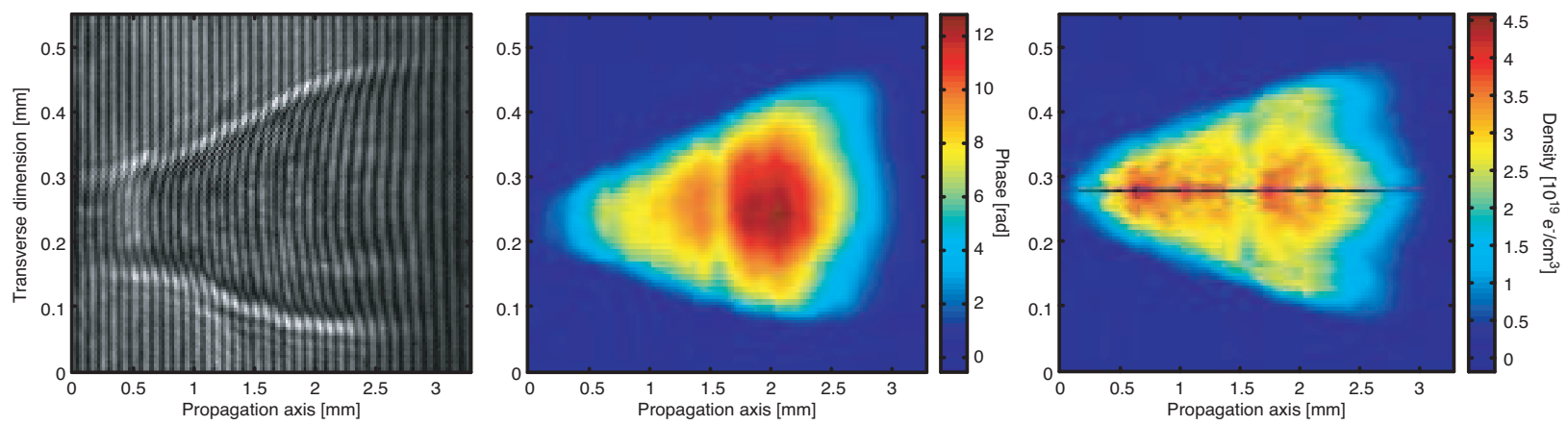

FIG. 2: Left, interferogram obtained for a back pressure of the gas jet of 600 psi Hydrogen. Center, phase map [radians] retrieved from Fourier analysis of the interferogram. Right, electron density map $\left[10^{19}\right.$ electrons $\left./ \mathrm{cm}^{3}\right]$ retrieved after symmetrization of the phase map and Abel inversion. 

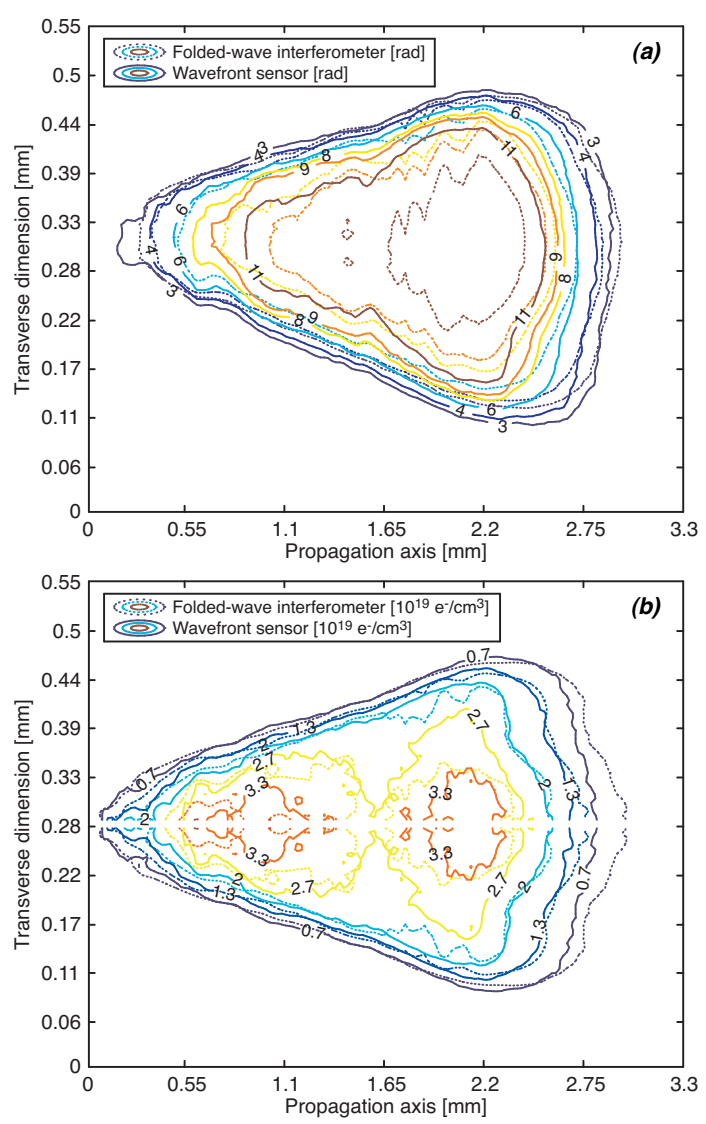

FIG. 3: Contour plots from wavefront sensor and interferometer of average phase maps (a) and average electron density maps (b) obtained at 600 psi Hydrogen. The average was performed on over 50 phase maps in both cases, wavefront sensor (solid lines) and folded-wave interferometer (dashed lines). 

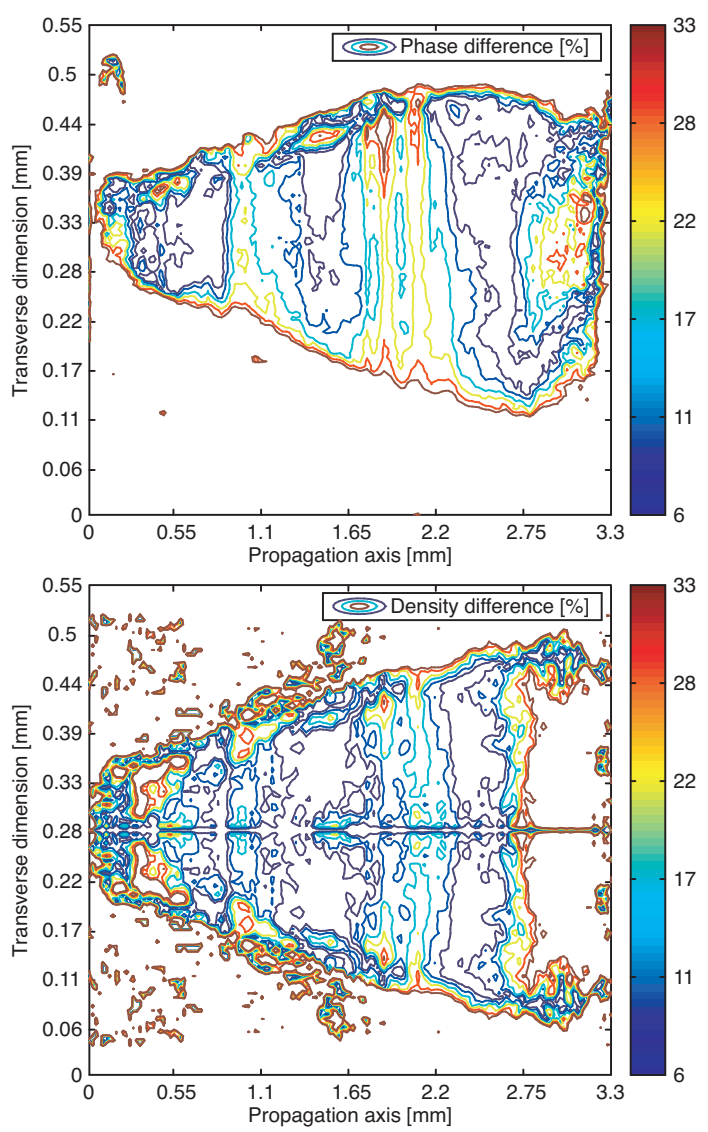

FIG. 4: Contour plots of the difference in percent between average phase maps (upper plot) and average electron density maps (lower plot) from wavefront sensing and folded-wave interferometry obtained at 600 psi Hydrogen. The average was performed on over 50 phase maps in both cases. In the region of interest, the difference between density measurements does not exceed $20 \%$. 


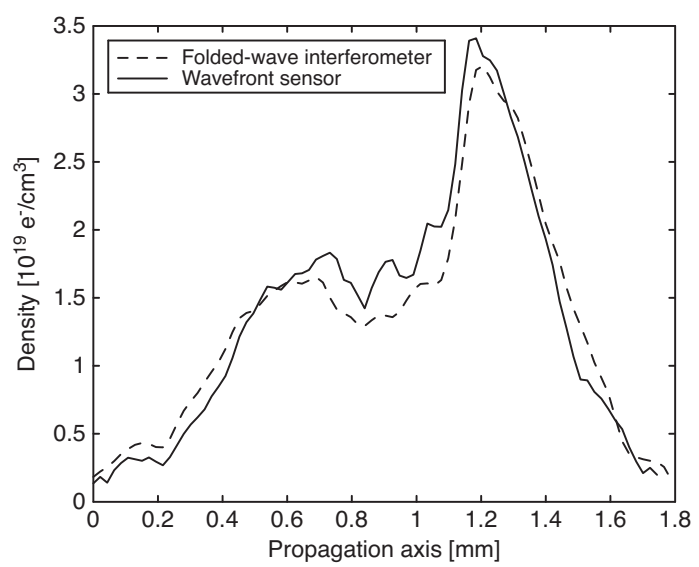

FIG. 5: Comparison between direct wavefront sensor measurements and folded-wave interferometry on a line out of the density maps obtained using a damaged gas jet nozzle (600 psi, Helium). Both measurements are capable of resolving the "shock" in the gas flow. 

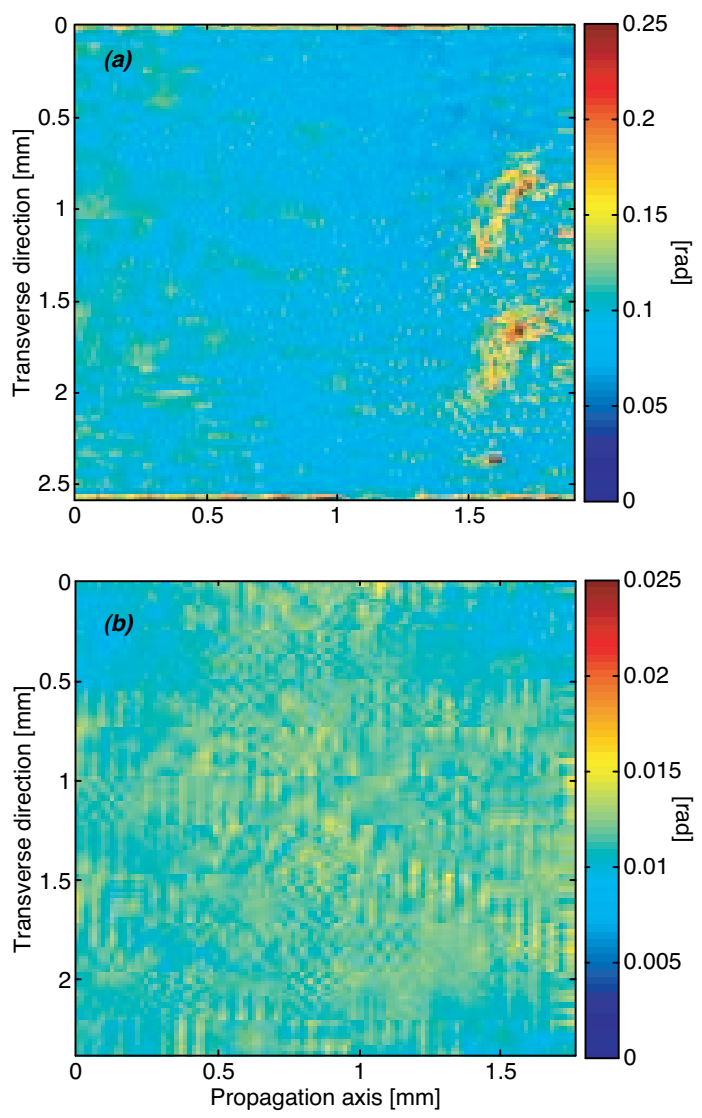

FIG. 6: Sensitivity measurements for folded-wave interferometry $(a)$ and wavefront sensing $(b)$. Each figure is the rms deviation of 188 phase maps obtained without plasma. Wavefront-sensorbased measurements are $\simeq 8.4$ times more sensitive and the noise is more homogeneously distributed. 


\begin{tabular}{|c|c|c|}
\cline { 2 - 3 } \multicolumn{1}{l|}{} & Folded-wave interferometer & Wavefront sensor \\
\hline $500 \mathrm{psi}$ & $2.06 \pm 0.25$ & $2.26 \pm 0.25$ \\
\hline $600 \mathrm{psi}$ & $2.43 \pm 0.30$ & $2.56 \pm 0.26$ \\
\hline $700 \mathrm{psi}$ & $2.69 \pm 0.32$ & $2.56 \pm 0.27$ \\
\hline
\end{tabular}

TABLE I: Comparison between direct wavefront sensor measurements and folded-wave interferometry for three different pressures. Values correspond to average and $r m s$ shot-to-shot deviation of the phase maps, and are indicated in $10^{19} e^{-} / \mathrm{cm}^{3}$. 